\title{
Risk factors for surgical site infection after intracranial electroencephalography monitoring for epilepsy in the pediatric population
}

\author{
Ying Meng, MD, ${ }^{1}$ Mathew R. Voisin, MD, ${ }^{1}$ Suganth Suppiah, MD,' Zamir Merali, MD,1 \\ Ali Moghaddamjou, MD, ${ }^{1}$ Naif M. Alotaibi, MD, MSc, ${ }^{1}$ \\ Arbelle Manicat-Emo, RN(EC), MS, NP-Paediatrics, ${ }^{3}$ Shelly Weiss, MD, FRCP, ${ }^{2}$ Cristina Go, MD, ${ }^{2}$ \\ Blathnaid McCoy, MD, MSc, ${ }^{2}$ Elizabeth J. Donner, MD, FRCPC, ${ }^{2}$ and \\ James T. Rutka, MD, PhD, FRCSC1,3
}

'Division of Neurosurgery, University of Toronto; and Divisions of ${ }^{2}$ Neurology and ${ }^{3}$ Neurosurgery, Hospital for Sick Children, Toronto, Ontario, Canada

\begin{abstract}
OBJECTIVE Intracranial electroencephalography (iEEG) monitoring is an important method of identifying the seizure focus in patients with medically refractory epilepsy. While previous studies have demonstrated low rates of surgical complications, reported rates of surgical site infection (SSI) are highly variable. To date, no studies have specifically evaluated the patient or operative risk factors contributing to SSI. The goals of this study were to examine the rate of SSI after iEEG monitoring for epilepsy workup in pediatric patients and to determine the variables that might contribute to the development of SSI.
\end{abstract}

METHODS A retrospective analysis of hospital charts at the Hospital for Sick Children was performed for all patients who had undergone iEEG monitoring between 2000 and 2016. Univariate and multivariate analyses were performed to look for statistically significant variables in relation to SSI.

RESULTS Among 199 patients eligible for analysis, 8 (4.0\%) developed SSIs within a period ranging from 21 to 51 days postoperatively. Univariate analysis yielded 4 factors related to SSI: number of people present in the operating room on electrode insertion $(p=0.02)$, length of insertion surgery $(p=0.04)$, previous operation at the same surgical site $(p=$ $0.04)$, and number of depth electrodes inserted $(p=0.01)$. Multivariate analysis revealed that both the number of people present during the implant operation (OR $0.08,95 \% \mathrm{Cl} \mathrm{0.01-0.70)}$ and the number of depth electrodes inserted (OR $3.52,95 \% \mathrm{Cl} 1.44-8.59)$ independently contributed to SSI.

CONCLUSIONS This is the largest case series and the first comprehensive review of both patient and operative risk factors in the development of SSI from iEEG monitoring in a pediatric population. The authors' institution had a lower rate of infection than those in most other studies, which could be explained by their protocol of administering intravenous antibiotics perioperatively and post-implant removal antibiotics for 14 days. The authors found a correlation between SSI and the number of people present during the implant operation, as well as the number of depth electrodes; both may contribute to breaks in sterility.

https://thejns.org/doi/abs/10.3171/2018.1.PEDS17476

KEYWORDS intracranial electroencephalography; surgical site infections; safety; epilepsy; pediatric neurosurgery; risk factors

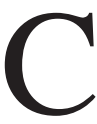
HILDREN with medically refractory epilepsy may benefit from early resection of the epileptogenic zone. In a subset of patients, noninvasive epilepsy assessment, such as MRI and surface electroencephalography (EEG), cannot accurately identify the ictal onset zone and precisely guide resective surgery. Intracranial electroencephalography (iEEG) monitoring augments the noninvasive assessments. The monitoring procedure helps the epilepsy team-a multidisciplinary team of neurologists, neurological surgeons, and allied health profession-

ABBREVIATIONS CSF = cerebrospinal fluid; EEG = electroencephalography; iEEG = intracranial EEG; MEG = magnetoencephalography; OR = operating room; SSI = surgical site infection.

SUBMITTED September 5, 2017. ACCEPTED January 23, 2018.

INCLUDE WHEN CITING Published online April 6, 2018; DOI: 10.3171/2018.1.PEDS17476. 
als involved in the assessment and management of children with epilepsy-evaluate patients who require more precise delineation of the ictal onset zone and mapping of the surrounding functional areas.

A combination of grid, strip, and depth electrodes are implanted near the suspected ictal onset zone with the wires connecting the electrodes tunneled underneath the skin. These intracranial electrodes can remain in place for a period ranging from days to weeks to monitor seizure activity. Multiple retrospective studies have documented a relatively low rate of complications with $\mathrm{EEEG}$, which is outweighed by the benefits of seizure localization. ${ }^{5,8,11,21}$ Surgical site infection (SSI), one such complication, occurs at a highly variable rate of between $0 \%$ and $15 \%$ in the pediatric population (Table 1). Such infections include intracranial infection, osteomyelitis, wound infection, and meningitis. In a recent meta-analysis of 2542 adult patients who underwent invasive EEG monitoring, the rate of SSI was $2.3 \% .^{1}$ However, a detailed examination of patient and operative factors that may be related to the occurrence of SSIs in pediatric patients undergoing iEEG monitoring has not been performed. Therefore, we conducted a retrospective analysis of the rate of SSI and the influencing factors by using the epilepsy database at our institution.

\section{Methods}

We identified consecutive patients who had undergone extraoperative iEEG monitoring at the Hospital for Sick Children in the period between 2000 and 2016. Data were de-identified after extraction from electronic patient records as well as scanned and categorized documents. Characteristics of the patients and their procedures (age, length of hospital stay, length of operation) were collected. We also noted the number of people present in the operating theater, which had been logged by the circulating nurse. The duration of the surgery was obtained from the $\log$ and recorded as the time between incision and closure. Furthermore, we defined an SSI as any postoperative infection involving the wound, skull, or central nervous system (meningitis, epidural abscess, osteomyelitis). The study was approved by the Research Ethics Board at the Hospital for Sick Children, and individual patient consent was waived.
An interdisciplinary epilepsy team assesses patients with intractable epilepsy refractory to multiple trials of anticonvulsant therapy for iEEG monitoring. Prior workup includes scalp EEG, video-EEG monitoring, neuroimaging (for example, MRI/functional MRI and magnetoencephalography [MEG]), and formal neuropsychological and language testing. Candidates for epilepsy surgery with invasive monitoring follow a standardized schedule. They are typically admitted on a Monday for implantation of the electrodes by a neurosurgeon with contributions from the epilepsy team and electrophysiologist in the operating room (OR). Patients undergo antiseptic hair wash the night before the neurosurgical procedure. They receive a preoperative dose of intravenous cefazolin, which is redosed every 4 hours as needed during the procedure. Steroids are not routinely given perioperatively. Based on seizure symptomatology and EEG and MEG findings, a customsized subdural grid of EEG electrodes and strip electrodes is placed over the surface of the brain. Depth electrodes are placed with stereotactic guidance if indicated. The implants are then sutured in place to the dura, and wires are individually tunneled through the scalp away from the incision. Purse-string knots are tied at the exit wounds to minimize the leakage of cerebrospinal fluid (CSF). The dura is closed using a large porcine graft, and the bone flap is replaced and hinged for the insertion procedure. Lastly, special attention is paid to making a full, secure head dressing to further protect the surgical site.

The patient is admitted to the critical care unit overnight for observation before transfer to the neurosurgery ward. A skull radiograph and head CT are obtained to verify grid placement. Patients receive intravenous vancomycin and cefotaxime for the entire period the electrodes are in situ. In most cases, a decision about resection is made in 4 days. However, implants have been left in place longer if there are insufficient data. After explantation of the electrodes, the patient receives an additional course of intravenous cloxacillin, which is transitioned to oral cephalexin for a total of 14 days. The antibiotic protocol was followed with near $100 \%$ compliance throughout the study period.

Statistical analysis of the relationship between variables and SSI was performed using IBM SPSS version 23 software (IBM Corp.). Chi-square and Fisher exact tests for categorical variables and the Mann-Whitney U-test for

TABLE 1. Rates of SSI after IEEG in the pediatric literature since 2000

\begin{tabular}{|c|c|c|c|}
\hline Authors \& Year & No. of Patients & Type of Electrodes & Infection Rate \\
\hline $\begin{array}{l}\text { Blauwblomme et al., } \\
\quad 2011\end{array}$ & 95 & $\begin{array}{l}1 \text { subdural grid per patient, }>1 \text { subdural strip per patient (mean 2, range 1-5), depth electrode } \\
\text { in } 31 \text { patients }\end{array}$ & 14.9 \\
\hline Musleh et al., 2006 & 29 & 1 subdural grid per patient, 24 depth electrodes total & 6.8 \\
\hline $\begin{array}{l}\text { Johnston et al., } \\
\quad 2006\end{array}$ & 112 & $\begin{array}{l}85 \text { patients had subdural grid \& strips, } 32 \text { had strips only, } 5 \text { had grids only, } 4 \text { had additional } \\
\text { depth electrodes }\end{array}$ & 2.4 \\
\hline Bauman et al., 2005 & 15 & 1 subdural grid per patient, depth electrode in 3 patients, strip electrodes in minority of patients & 6.7 \\
\hline Roth et al., 2014 & 161 & No exact info; patients had some combination of subdural strips, grids, \& often depth electrodes & 6.3 \\
\hline Onal et al., 2003 & 35 & 1 subdural grid per patient, 34 patients had strip electrodes, 17 had depth electrodes & 8.57 \\
\hline Simon et al., 2003 & 64 & 62 patients had subdural grid \& strips, 2 patients had stereotactic depth electrodes & 15.6 \\
\hline Bruce \& Bizzi, 2000 & 85 & $\begin{array}{l}\text { No exact info; most patients had } 1 \text { subdural grid \& 3-6 subdural strips, depth electrodes rarely } \\
\text { used }\end{array}$ & 5.8 \\
\hline
\end{tabular}


continuous variables were performed for univariate analysis. Significant predictors $(\mathrm{p}<0.10)$ were tested in a multivariable logistic regression to identify independent risk factors with $\mathrm{p}=0.05$, and the results were reported as an odds ratio with a $95 \%$ confidence interval.

\section{Results}

For the period between 2000 and 2016, we documented information on 199 consecutive patients who underwent iEEG. Their ages ranged from 1.5 to 18 years, with an average of 10.9 years (Table 2). In 26 patients, the procedure was a repeat operation at the same cranial site. Subdural grid electrodes were implanted in almost all of the patients (92.0\%). A majority of the patients also had strip (76.9\%) and depth (75.9\%) electrodes. Patients were monitored for 2-14 days, on average 4.5 days. All patients were followed up for at least 6 months. Seventeen patients (8.5\%) experienced operative infections of all types.

The workup for patients suspected to have an SSI included but was not limited to a wound swab and neuroimaging. When a collection was present, surgical drainage and washout were considered for source control and procuring a culture. Further management decisions were made in a multidisciplinary manner depending on the culture, the sensitivity, and the patient's overall health and social situation. While 17 patients $(8.5 \%)$ experienced postoperative infections, only 8 cases $(4.0 \%)$ involved the surgical site. These cases were documented between 21 and 51 days after surgery. Five patients had superficial wound infections only, which resolved with a course of antibiotics in all 5 cases. The specifics of each SSI are listed in Table 3.

We noted a variety of microorganisms isolated, including a case of Candida albicans and 2 cases of Pseudomonas aeruginosa. A piece of the dural graft was also sent for culture just prior to insertion; in 2 cases, the cultures grew Propionibacterium acnes and Staphylococcus epidermidis. We did not alter the standard postoperative antibiotic regimen for these 2 patients. Neither of them demonstrated overt signs of infection on follow-up. Finally, other reasons for postoperative infection included pneumonia, urinary tract infection, gastroenteritis, and parotitis. Four patients had unexplained fevers after surgery that improved with supportive care.

Results from the univariate analysis are summarized in Table 4 . We found 4 factors that significantly correlated with SSI: number of people at the insertion surgery $(\mathrm{p}=$ $0.02)$, length of insertion procedure $(\mathrm{p}=0.04)$, previous operation at the same surgical site $(p=0.04)$, and number of depth electrodes $(p=0.01)$. We dichotomized 2 variables: the number of people present at the insertion procedure at 15 and the length of the operation at 4 hours. The threshold for the former variable was estimated at 15 to include the surgical, anesthesia, electrophysiology, epilepsy, and nursing team, and the latter was established at 4 hours given that the number of cases without SSI fell equally on both sides. Additionally, 4 hours is a practical delineation as it is the length of a typical insertion procedure. The average duration of our insertion surgeries was 4.4 hours. We chose not to dichotomize other factors, such
TABLE 2. Summary of characteristics of 199 patients who underwent iEEG monitoring

\begin{tabular}{lc}
\hline \multicolumn{1}{c}{ Characteristic } & Value \\
\hline Age in yrs & $10.9 \pm 4.8$ \\
\hline Duration of iEEG monitoring in days & $4.5 \pm 1.7$ \\
\hline Length of hospital stay in days & $12.0 \pm 5.6$ \\
\hline No. of patients w/ & \\
Grid electrode & $183(92.0 \%)$ \\
Strip electrode & $153(76.9 \%)$ \\
Depth electrode & $151(75.9 \%)$ \\
\hline Mean no. of & \\
Strip electrodes placed & $2.3 \pm 1.9$ \\
Depth electrodes inserted & $2.2 \pm 0.9$ \\
\hline No. of people at & \\
Insertion of electrodes & $15.9 \pm 4.2$ \\
Removal of electrodes & $15.3 \pm 5.1$ \\
\hline Duration of insertion surgery in hrs & $4.4 \pm 1.2$ \\
\hline Patients w/ prior operation at the same site & $26(13.1 \%)$ \\
\hline Mean no. of anticonvulsants & $2.4 \pm 0.9$ \\
\hline Patients w/ positive dural graft culture & 2 \\
\hline Postop infection & $17(8.5 \%)$ \\
Pneumonia & $6(3.0 \%)$ \\
Gastroenteritis & $1(0.5 \%)$ \\
Urinary tract infection & $1(0.5 \%)$ \\
Parotitis & $1(0.5 \%)$ \\
\hline SSI & $8(4.0 \%)$ \\
No. of days after surgery & $33.7 \pm 14.8$ \\
Intracranial infection & $3(1.5 \%)$ \\
Meningitis only & 0 \\
Wound infection only & $5(2.5 \%)$ \\
\hline Cases requiring surgical debridement & $4(2.0 \%)$ \\
\hline
\end{tabular}

Values are expressed as the mean \pm standard deviation or as number of patients (\%).

as the number of surface and depth electrodes, given the difficulty in determining a clinically meaningful value. The association between SSI and duration of iEEG monitoring or length of the hospital stay was not statistically significant.

We also performed a multivariable logistic regression analysis of the factors previously identified to potentially associate with SSI $(p<0.10)$ to determine the independent risk factors for SSI (Table 5). The number of people present at the implantation operation and the number of depth electrodes independently contributed to SSI. Fifteen or fewer people at the implantation surgery had an OR of 0.08 for SSI (95\% CI 0.01-0.70). The number of depth electrodes was found to have a strong association, with an OR of 3.52 (95\% CI 1.44-8.59). Finally, a first-time operation did not reach statistical significance, with an OR of 0.17 (95\% CI 0.03-1.04).

\section{Discussion}

Evidence suggests that earlier epilepsy surgery in children leads to improved long-term neurological and functional outcomes. ${ }^{4,10,20}$ A longitudinal study of children who 
TABLE 3. Characteristics of SSI cases

\begin{tabular}{ccclll}
\hline Case No. & Age $(\mathrm{yrs})$ & No. of Days From Surgery & Type of Infection & Organism & Treatment \\
\hline 1 & 15 & 21 & Wound & Candida albicans & Antimicrobial \\
\hline 2 & 17 & 10 & Wound & Pseudomonas aeruginosa & Antimicrobial \\
\hline 3 & 16 & NA & Wound & NA & Antimicrobial \\
\hline 5 & 6 & 36 & Wound & Pseudomonas aeruginosa & Antimicrobial \\
\hline 6 & 7 & 28 & Wound & Moraxella catarrhalis & Antimicrobial plus surgical washout \\
\hline 7 & 9 & 46 & Intracranial & Negative growth & Antimicrobial plus surgical washout \\
\hline 8 & 3 & 51 & Intracranial & Staphylococcus aureus & Antimicrobial plus surgical washout \\
\hline
\end{tabular}

$\mathrm{NA}=$ not available

underwent epilepsy surgery versus nonsurgical matched controls showed significantly improved seizure control and social adaptation. ${ }^{20}$ The developmental trajectories in terms of memory and language in the surgical group were positive and significantly different from those in the control group. Intracranial EEG monitoring, in addition to less invasive methods, such as functional MRI, MEG, and surface EEG, is an invaluable tool in localizing the epileptogenic focus. ${ }^{11}$ As various centers accumulate experience with iEEG monitoring, the goal of minimizing complications, such as postoperative infections that may have longterm effects on a child's health, moves to the forefront.

Our study is the largest retrospective single-center series on iEEG monitoring in children. We aimed to explore the rate of and the risk factors for postoperative SSI.

TABLE 4. Patient and operative risk factors for SSI in univariate analysis

\begin{tabular}{|c|c|c|c|}
\hline Factor & $\begin{array}{c}\text { Value w/ } \\
\text { SSI }\end{array}$ & $\begin{array}{c}\text { Value w/o } \\
\text { SSI }\end{array}$ & $\begin{array}{c}p \\
\text { Value }\end{array}$ \\
\hline Age in yrs & $10.8 \pm 5.2$ & $10.9 \pm 4.8$ & 0.94 \\
\hline \multicolumn{4}{|l|}{ Duration of iEEG monitoring } \\
\hline$\leq 4$ days & 7 & 156 & 0.67 \\
\hline$>4$ days & 1 & 35 & \\
\hline Length of hospital stay in days & $13.1 \pm 5.9$ & $11.9 \pm 5.6$ & 0.84 \\
\hline \multicolumn{4}{|l|}{ No. of people at insertion surgery } \\
\hline$\leq 15$ people & 1 & 102 & 0.02 \\
\hline$>15$ people & 7 & 89 & \\
\hline \multicolumn{4}{|l|}{ No. of people at removal } \\
\hline$\leq 15$ people & 4 & 105 & 0.78 \\
\hline$>15$ people & 4 & 86 & \\
\hline \multicolumn{4}{|l|}{ Length of insertion surgery } \\
\hline$\leq 4 \mathrm{hrs}$ & 1 & 96 & 0.04 \\
\hline$>4 \mathrm{hrs}$ & 7 & 95 & \\
\hline Grid electrodes placed & 7 & 160 & 0.84 \\
\hline No. of strip electrodes placed & $1.8 \pm 2.6$ & $1.8 \pm 1.9$ & 0.63 \\
\hline No. of depth electrodes inserted & $2.8 \pm 0.8$ & $1.6 \pm 1.2$ & 0.01 \\
\hline Prior operation at the same site & 3 & 23 & 0.04 \\
\hline No. of anticonvulsants & $2.3 \pm 0.9$ & $2.4 \pm 0.8$ & 0.85 \\
\hline
\end{tabular}

Values are expressed as the mean \pm standard deviation or as number of patients, unless indicated otherwise. Boldface type indicates statistical significance.
While a handful of case series of iEEG operations have documented rates of infection, our study is the first to examine a variety of patient and operative variables and their association with the risk of SSI. In our analysis, we discovered risk factors that are congruent with those in the existing literature, as well as factors that have rarely been examined in previous studies.

Notably, we observed an SSI rate of $4.0 \%$ in our cohort, which falls in the lower end of what has been reported in the literature $(0 \%-15 \%)$. Given the size of our study cohort, we believe that a $4.0 \%$ infection rate more accurately reflects a large center with experienced surgeons and epilepsy team. Another possible explanation for the lower infection rate is our institution's surgical protocol of prescribing intravenous antibiotics while the electrodes remain in place, as well as prophylactic antibiotics for 2 weeks after electrode removal. Some institutions reporting a higher rate of SSI did not have a policy of prophylactic antibiotic use during the monitoring period. ${ }^{5}$

A previous retrospective study of iEEG monitoring suggested that age, number of exiting wires, duration of monitoring, and grid size were associated with rates of SSI. ${ }^{22}$ On the other hand, a case series of 67 iEEG procedures in children found no statistically significant correlation between the length of time a patient is on perioperative antibiotics, duration of monitoring, or CSF leakage and SSI. ${ }^{21}$ A potential explanation for inconsistencies among different studies may be the relatively low rate of SSI. In our cohort, univariate analysis identified 4 factors that were significantly correlated with infection risk: repeat operation at same site, number of people at insertion surgery, duration of electrode insertion surgery, and number of depth electrodes. Of these variables, the presence of more than 15 people in the operating theater and the number of

TABLE 5. Results of multivariate analysis to determine independent risk factors for SSI

\begin{tabular}{ll}
\hline \multicolumn{1}{c}{ Factor } & \multicolumn{1}{c}{ OR $(95 \% \mathrm{Cl})$} \\
\hline$\leq 15$ people at insertion procedure & $0.08(0.01-0.70)^{*}$ \\
\hline Insertion procedure $\leq 4 \mathrm{hrs}$ & $0.21(0.02-1.95)$ \\
\hline No. of depth electrodes & $3.52(1.44-8.59)^{*}$ \\
\hline First-time operation & $0.17(0.03-1.04)$ \\
\hline * Denotes $p<0.05$. &
\end{tabular}


depth electrodes were found to be independent risk factors for SSI. Although the correlation between repeat operation and infection did not reach statistical significance in our multivariable analysis, reoperation has been reported as a significant predictor of postoperative infection..$^{15}$

During an electrode insertion surgery, a multidisciplinary team including neurosurgeons, surgical nurses, neurologists, electrophysiologists, and other support staff is present to ensure correct placement of the electrodes. Students are sometimes present to observe for educational purposes. These individuals are entering and leaving the OR sporadically, leading to multiple openings of the OR doors throughout the surgery. Operating theater quality control studies have demonstrated that the air quality is degraded by an increased number of door openings. ${ }^{2,14,18}$ Furthermore, the disruptions in air quality lead to an increased bacterial burden on surgical sites and higher rates of infection. ${ }^{7}$ A multidisciplinary team is essential for iEEG operations; however, one potential strategy for protecting sterility may be to limit the entrances and exits to specified times during the operation to maximize efficiency and minimize air quality disruptions. Another strategy is to limit the personnel allowed to enter the OR to a core group consisting of the operating team, neurologists, and neurophysiologists. Observers may view the procedure through a secured video stream or a viewing area outside the OR.

It is interesting that the number of depth electrodes was the strongest risk factor, whereas similar variables, such as the number of strip electrodes, were not significant. Previous retrospective series have presented mixed evidence regarding the association between the number of electrode contacts and postoperative complications. ${ }^{8,9,11,16}$ While these studies did not parse out the individual types of complications, the increased implant size or increased contact with the brain surface may explain the increased risk to the patient. A retrospective analysis of stereotactically inserted depth electrodes documented very low rates of SSI ( $0.2 \%$ meningitis, $0.2 \%$ local cutaneous infection). ${ }^{6}$ Although our study did not identify duration of surgery as an independent risk factor for SSI, the length of the operation and the duration of anesthesia have been shown to be risk factors for SSI in both adult and pediatric neurosurgery. ${ }^{13,19}$ We noted on average a 30 -minute increase in surgery time with the insertion of depth electrodes. As a result, the extra time required to place the depth electrodes may account for the significance of the length of surgery. Finally, we did not find any correlation between the duration of monitoring and the rate of SSI. This suggests that if the data collected within a standard 4-day monitoring are insufficient (for example, the patient did not have a seizure), it is reasonable to extend the duration of monitoring. Note that some evidence suggests that monitoring more than 14 days is positively associated with SSI. ${ }^{22}$

Although our study reports the infection risk in a large cohort, the study is limited by its retrospective nature. For instance, data collected from chart review are subject to recall bias, as in the instance of patient compliance with antibiotics after discharge from the hospital. Additionally, given the number of missing data points for the preoperative white blood cell count, we were unable to include this information for analysis. Our model did not include information on the length of the removal surgery, presence of CSF leakage, or quality of the wound or wound care. Patient factors such as cognitive deficit could also influence the state of the wound after surgery.

\section{Conclusions}

Over the last 16 years, our rate of postoperative SSI was $4.0 \%$. We found that the duration of the insertion procedure, a history of surgery at the same location, the number of depth electrodes, and the number of people present in the OR were potential risk factors for SSI. This is the first time that the association between patient and operative variables and SSI has been closely analyzed for pediatric patients who had undergone iEEG monitoring. Extensive studies have been done on shunt procedures in the pediatric population, yielding a multicenter protocol that has been shown to decrease complications. ${ }^{12}$ Although iEEG implants are temporary, a paradigm similar to the multicenter protocol may benefit patients undergoing monitoring for diagnostic purposes. Therefore, the results of our analysis will inform improvements in the operative protocol, electrode technology, and surgical techniques to minimize postoperative infections.

\section{References}

1. Arya R, Mangano FT, Horn PS, Holland KD, Rose DF, Glauser TA: Adverse events related to extraoperative invasive EEG monitoring with subdural grid electrodes: a systematic review and meta-analysis. Epilepsia 54:828-839, 2013

2. Babkin Y, Raveh D, Lifschitz M, Itzchaki M, Wiener-Well $\mathrm{Y}$, Kopuit P, et al: Incidence and risk factors for surgical infection after total knee replacement. Scand J Infect Dis 39:890-895, 2007

3. Bauman JA, Feoli E, Romanelli P, Doyle WK, Devinsky O, Weiner HL: Multistage epilepsy surgery: safety, efficacy, and utility of a novel approach in pediatric extratemporal epilepsy. Neurosurgery 56:318-334, 2005

4. Benifla M, Rutka JT, Otsubo H, Lamberti-Pasculli M, Elliott I, Sell E, et al: Long-term seizure and social outcomes following temporal lobe surgery for intractable epilepsy during childhood. Epilepsy Res 82:133-138, 2008

5. Blauwblomme T, Ternier J, Romero C, Pier KST, D’Argenzio L, Pressler R, et al: Adverse events occurring during invasive electroencephalogram recordings in children. Neurosurgery 69 (2 Suppl Operative):ons169-ons175, 2011

6. Bourdillon P, Ryvlin P, Isnard J, Montavont A, Catenoix H, Mauguière F, et al: Stereotactic electroencephalography is a safe procedure, including for insular implantations. World Neurosurg 99:353-361, 2017

7. Brohus H, Balling KD, Jeppesen D: Influence of movements on contaminant transport in an operating room. Indoor Air 16:356-372, 2006

8. Bruce DA, Bizzi JW: Surgical technique for the insertion of grids and strips for invasive monitoring in children with intractable epilepsy. Childs Nerv Syst 16:724-730, 2000

9. Hamer HM, Morris HH, Mascha EJ, Karafa MT, Bingaman WE, Bej MD, et al: Complications of invasive video-EEG monitoring with subdural grid electrodes. Neurology 58:97103,2002

10. Hosoyama H, Matsuda K, Mihara T, Usui N, Baba K, Inoue Y, et al: Long-term outcomes of epilepsy surgery in 85 pediatric patients followed up for over 10 years: a retrospective survey. J Neurosurg Pediatr 19:606-615, 2017 
11. Johnston JM Jr, Mangano FT, Ojemann JG, Park TS, Trevathan E, Smyth MD: Complications of invasive subdural electrode monitoring at St. Louis Children's Hospital, 19942005. J Neurosurg 105 (5 Suppl):343-347, 2006

12. Kestle JRW, Holubkov R, Douglas Cochrane D, Kulkarni AV, Limbrick DD Jr, Luerssen TG, et al: A new Hydrocephalus Clinical Research Network protocol to reduce cerebrospinal fluid shunt infection. J Neurosurg Pediatr 17:391-396, 2016

13. Korinek AM, Laisne MJ, Achou C, Bromberg N, Dagreou F, Debout J, et al: Risk factors for neurosurgical site infections after craniotomy: a prospective multicenter study of 2944 patients. Neurosurgery 41:1073-1081, 1997

14. Lynch RJ, Englesbe MJ, Sturm L, Bitar A, Budhiraj K, Kolla $S$, et al: Measurement of foot traffic in the operating room: implications for infection control. Am J Med Qual 24:45 52, 2009

15. Musleh W, Yassari R, Hecox K, Kohrman M, Chico M, Frim D: Low incidence of subdural grid-related complications in prolonged pediatric EEG monitoring. Pediatr Neurosurg 42:284-287, 2006

16. Onal C, Otsubo H, Araki T, Chitoku S, Ochi A, Weiss S, et al: Complications of invasive subdural grid monitoring in children with epilepsy. J Neurosurg 98:1017-1026, 2003

17. Roth J, Carlson C, Devinsky O, Harter DH, Macallister WS, Weiner HL: Safety of staged epilepsy surgery in children. Neurosurgery 74:154-162, 2014

18. Scaltriti S, Cencetti S, Rovesti S, Marchesi I, Bargellini A, Borella P: Risk factors for particulate and microbial contamination of air in operating theatres. J Hosp Infect 66:320 326, 2007

19. Sherrod BA, Arynchyna AA, Johnston JM, Rozzelle CJ, Blount JP, Oakes WJ, et al: Risk factors for surgical site infection following nonshunt pediatric neurosurgery: a review of 9296 procedures from a national database and compari- son with a single-center experience. J Neurosurg Pediatr 19:407-420, 2017

20. Sibilia V, Barba C, Metitieri T, Michelini G, Giordano F Genitori L, et al: Cognitive outcome after epilepsy surgery in children: a controlled longitudinal study. Epilepsy Behav 73:23-30, 2017

21. Simon SL, Telfeian A, Duhaime AC: Complications of invasive monitoring used in intractable pediatric epilepsy. Pediatr Neurosurg 38:47-52, 2003

22. Wiggins GC, Elisevich K, Smith BJ: Morbidity and infection in combined subdural grid and strip electrode investigation for intractable epilepsy. Epilepsy Res 37:73-80, 1999

\section{Disclosures}

The authors report no conflict of interest concerning the materials or methods used in this study or the findings specified in this paper.

\section{Author Contributions}

Conception and design: Meng, Manicat-Emo, Donner, Rutka Acquisition of data: Meng, Voisin, Merali, Moghaddamjou, Manicat-Emo. Analysis and interpretation of data: Meng, Voisin, Suppiah, Merali, Moghaddamjou, Go, McCoy. Drafting the article: Meng, Suppiah. Critically revising the article: Meng, Voisin, Suppiah, Alotaibi, Weiss, Go, McCoy, Rutka. Reviewed submitted version of manuscript: all authors. Approved the final version of the manuscript on behalf of all authors: Meng. Statistical analysis: Meng, Voisin, Suppiah. Study supervision: Rutka.

\section{Correspondence}

Ying Meng: Hospital for Sick Children, Toronto, ON, Canada. ying.meng@utoronto.ca. 